\title{
Linear Adiabatic Theory. Exponential Estimates
}

\author{
G. Nenciu \\ Institut für Theoretische Physik, Universität Zürich, Schönberggasse 9, \\ CH-8001 Zürich, Switzerland *
}

Received March 4, 1992; in revised form August 10, 1992

\begin{abstract}
A general adiabatic expansion is written down. The basic result is that, under appropriate smoothness conditions, the adiabatic estimations can be pushed up to exponential order. The results imply exponential estimates not only for hamiltonians analytic in a neighbourhood of the real axis but also for hamiltonians which (in an appropriate sense) beong to Gevrey classes.
\end{abstract}

\section{The Problem, Heuristics, Results}

For definiteness in this section we shall consider unitary evolutions in Hilbert spaces. However, the main results in Sect. 2 hold true in a more general context.

Consider the evolution, $U_{\varepsilon}\left(s, s_{0}\right)$, given by

$$
i \varepsilon \frac{d}{d s} U_{\varepsilon}\left(s, s_{0}\right)=H(s) U_{\varepsilon}\left(s, s_{0}\right) ; \quad U_{\varepsilon}\left(s_{0}, s_{0}\right)=1
$$

in the limit $\varepsilon \rightarrow 0$. Since, as it is well known, (1.1) is hard to integrate, one can pose the problem to obtain information about $U_{\varepsilon}\left(s, s_{0}\right)$ without actually integrating (1.1). Our strategy is to proceed in two steps:

I) Find out (almost) invariant subspaces, $\mathscr{K}(s ; \varepsilon)$, under the evolution $U_{\varepsilon}\left(s, s_{0}\right)$, i.e.

$$
U_{\varepsilon}\left(s, s_{0}\right) \mathscr{K}\left(s_{0} ; \varepsilon\right) \cong \mathscr{K}(s ; \varepsilon) .
$$

II) Integrate the evolution equation restricted to the subspaces $\mathscr{K}(s ; \varepsilon)$. Let us mention that this step is trivial if (as it is the case in many physical applications) $\operatorname{dim} \mathscr{K}(s ; \varepsilon)=1$.

In the rest of this section we shall describe, at the heuristic level, our procedure to solve I) and II) and outline the relation of our results with some of the previously

\footnotetext{
* Permanent address: Dept. Theor. Phys., University of Bucharest, PO Box MG 11, R-76900 Bucharest, Romania
} 
known results. The precise formulations and the proofs are contained in Sect. 2 and 3.

If the subspaces $\mathscr{K}(s ; \varepsilon)$ are described by the projection operators, $P_{\varepsilon}(s)$, i.e., $\mathscr{K}(s ; \varepsilon)=P_{\varepsilon}(s) \mathscr{H}$, the condition (1.2) becomes the (approximate) intertwining property

$$
P_{\varepsilon}(s) U_{\varepsilon}\left(s, s_{0}\right) \cong U_{\varepsilon}\left(s, s_{0}\right) P_{\varepsilon}\left(s_{0}\right)
$$

or equivalently

$$
P_{\varepsilon}(s) \cong U_{\varepsilon}\left(s, s_{0}\right) P_{\varepsilon}\left(s_{0}\right) U_{\varepsilon}\left(s, s_{0}\right)^{-1} .
$$

Differentiating (1.3) one obtains the well known Heisenberg equation of motion

$$
i \varepsilon \frac{d}{d s} P_{\varepsilon}(s) \cong\left[H(s), P_{\varepsilon}(s)\right] \text {. }
$$

Notice that the evolution operator does not appear in (1.4). To solve step I. means to find solutions of (1.4) satisfying also the projection property

$$
P_{\varepsilon}(s)=P_{\varepsilon}(s)^{2} .
$$

The simplest thing is to try the "ansatz" (for the moment at the level of formal series)

$$
P_{\varepsilon}(s)=\sum_{j=0}^{\infty} E_{j}(s) \varepsilon^{j} .
$$

Inserting (1.6) into (1.4) and (1.5) one obtains

$$
\begin{gathered}
{\left[H(s), E_{0}(s)\right]=0, \quad i \frac{d}{d s} E_{\jmath}(s)=\left[H(s), E_{j+1}(s)\right],} \\
E_{\jmath}(s)=\sum_{m=0}^{\jmath} E_{m}(s) E_{j-m}(s) .
\end{gathered}
$$

Suppose that for all $s, H(s)$ has an isolated bounded part, $\sigma_{0}(s)$, of the spectrum and let $P_{0}(s)$ be the spectral projection of $H(s)$ corresponding to $\sigma_{0}(s)$. Our first result is (under appropriate smoothness conditions on $H(s)$ ) an explicit recurrence formula for $E_{\jmath}(s)$ which solves (1.7), (1.8) (see Lemma 1 for precise formulation). For the needs of the discussion below let us mention the following property of $E_{\jmath}(s)$ : if outside $(-1,1), H(s)$ does not depend on $s$ then $E_{\jmath}(s)=0$ for all $s \notin(-1,1)$ and $j \geq 1$. Alternatively if $H(s)$ approaches limits as $s \rightarrow \pm \infty$ sufficiently fast then again $E_{j}(s) \rightarrow 0$ for $j \geq 1$ as $s \rightarrow \pm \infty$.

As it stands, the series in the r.h.s. of (1.6) is usually not convergent; one needs to truncate the series at some order and construct out of the partial sum (a finite polynomial cannot satisfy (1.5)!) a "bona fide" projection satisfying (1.4). For that, a control on the norms of $E_{j}(s)$ is needed. This control (see Lemma 3 ) is our main technical result. The choice of the truncation order is by a "optimal remainder" estimate. The outcome is a $P_{\varepsilon}(s)$ satisfying (1.5) and

$$
\left\|i \varepsilon \frac{d}{d s} P_{\varepsilon}(s)-\left[H(s), P_{\varepsilon}(s)\right]\right\| \leq \delta(s ; \varepsilon),
$$

where $\delta(s ; \varepsilon)$ depends upon the smoothness of $H(s)$ : if (in an appropriate sense, see Sect. 2) $H(s) \in \mathscr{C}^{k}$ then $\delta(s ; \varepsilon) \sim \varepsilon^{k}$; if $H(s)$ is holomorphic in a strip around real axis then $\delta(s ; \varepsilon) \sim \exp (-$ const $/ \varepsilon)$ and if $H(s)$ belongs to the Gevrey class of order 
$\alpha$ then $\delta(s ; \varepsilon) \sim \exp \left(-\right.$ const $\left./ \varepsilon^{1 / 1+\alpha}\right)$. The inequality (1.9) is our basic result. Few remarks are in order. First, the construction of $P_{\varepsilon}(s)$ is local in $s$ in the sense that no integrations over $s$ are involved and only $H(s)$ and its derivatives up to some finite order enter the formulae for $P_{\varepsilon}(s)$. In some loose sense the construction of $P_{\varepsilon}(s)$ is the analog of the Riesz formula which gives the spectral projection corresponding to an isolated part of the spectrum as a contour integral of the resolvent. Secondly, while as it is well known an expansion of the form

$$
U_{\varepsilon}\left(s, s_{0}\right)=U_{0}\left(s, s_{0}\right)+\varepsilon U_{1}\left(s, s_{0}\right)+\ldots
$$

does not exist, the evolution of subspaces (for appropriately chosen initial conditions) has a nice behaviour as $\varepsilon \rightarrow 0$. One can see the reason looking at the onedimensional subspace case. Let $\psi_{\varepsilon}(s)=U_{\varepsilon}\left(s, s_{0}\right) \psi\left(s_{0}\right)$. Even if an expansion $\psi_{\varepsilon}(s)=\psi_{0}(s)+\varepsilon \psi_{1}(s)+\ldots$ does not exist, a nice expansion for the corresponding projection $\left|\psi_{\varepsilon}(s)\right\rangle\left\langle\psi_{\varepsilon}(s)\right|$ might still exist if all the singular behaviour of $\psi_{\varepsilon}(s)$ is concentrated in a phase factor which cancels out in $\left|\psi_{\varepsilon}(s)\right\rangle\left\langle\psi_{\varepsilon}(s)\right|$. Notice the similarity with the behaviour of the wave function in the semiclassical limit as given by the WKB ansatz.

Using the fact that $U_{\varepsilon}\left(s, s_{0}\right)$ is unitary one can obtain at once from (1.9)

$$
\left\|P_{\varepsilon}(s)-U_{\varepsilon}\left(s, s_{0}\right) P_{\varepsilon}\left(s_{0}\right) U_{\varepsilon}\left(s, s_{0}\right)^{*}\right\| \leq \varepsilon^{-1} \int_{s_{0}}^{s} \delta(u ; \varepsilon) d u
$$

and

$$
\left\|\left(1-P_{\varepsilon}(s)\right) U_{\varepsilon}\left(s, s_{0}\right) P_{\varepsilon}\left(s_{0}\right)\right\| \leq \varepsilon^{-1} \int_{s_{0}}^{s} \delta(u ; \varepsilon) d u
$$

which e.g. in the analytic case is an adiabatic theorem to exponential order for all $s, s_{0} \in \mathbf{R}$. Notice that while the usual adiabatic theory estimates the transition probabilities after infinite time the above result estimates the transition for all times. The connection with the adiabatic theorem in its standard form [ASY, BF, K1, N1] is made observing that (1.11) implies

$$
\begin{aligned}
& \left\|\left(1-P_{0}(s)\right) U_{\varepsilon}\left(s, s_{0}\right) P_{0}\left(s_{0}\right)\right\| \\
& \quad \leq\left\|P_{\varepsilon}(s)-P_{0}(s)\right\|+\left\|P_{\varepsilon}\left(s_{0}\right)-P_{0}\left(s_{0}\right)\right\|+\varepsilon^{-1} \int_{s_{0}}^{s} \delta(u ; \varepsilon) d u .
\end{aligned}
$$

Actually (1.12) gives more than the standard adiabatic theorem since the r.h.s. of (1.12) is $O(\varepsilon)$ for all $s$ such that $\int_{s_{0}}^{s} \delta(u ; \varepsilon) d u \leq \varepsilon^{2}$ and these intervals can be exponentially long if e.g. $H(s)$ is analytic in a strip around the real axis. Also (1.12) contains all variants of the "adiabatic theorem of arbitrary order" starting with the result of Lenard [Le] and ending with the result in [KS] and the recent exponential estimate in [JP1]. Indeed for $s, s_{0} \notin \operatorname{supp} \frac{d}{d s} H(s), P_{\varepsilon}(s)=P_{0}(s)$ so that

$$
\left\|\left(1-P_{0}(s)\right) U_{\varepsilon}\left(s, s_{0}\right) P_{0}\left(s_{0}\right)\right\| \leq \varepsilon^{-1} \int_{s_{0}}^{s} \delta(u ; \varepsilon) d u .
$$


For the case when $H(s)$ approaches limits as $s \rightarrow \pm \infty$ sufficiently fast (1.13) becomes

$$
\lim _{s \rightarrow \infty, s_{0} \rightarrow-\infty}\left\|\left(1-P_{0}(s)\right) U_{\varepsilon}\left(s, s_{0}\right) P_{0}\left(s_{0}\right)\right\| \leq \varepsilon^{-1} \int_{-\infty}^{\infty} \delta(u ; \varepsilon) d u .
$$

Let us notice that while all the above results are upper bounds of the adiabatic transitions, in some simple cases it has been possible to compute the asymptotics as $\varepsilon \rightarrow 0$ of the adiabatic transitions ([B1, JKP, JMP, H1] and references therein; for the related problem of adiabatic invariants for the harmonic oscillator see $[\mathrm{HK}]$ ). Also, our results apply only to isolated parts of the spectrum; for results concerning crossing eigenvalues and dense point spectra see [H2, AHS].

The existence of subspaces invariant up to the order $\varepsilon^{k}, k=1,2, \ldots$ has been pointed out for the first time (according to our best knowledge) by Garrido [G]. A rigorous procedure for constructing $P_{k}(s ; \varepsilon)$ such that $P_{k}(s ; \varepsilon) \mathscr{H}$ are invariant up to errors of order $\varepsilon^{k+1}$ has been given by Nenciu [N2] (see also [N3, NR1] and for a slightly different procedure [JP2]); the procedure in this paper and the procedures in the papers quoted above are related: the expansions in powers of $\varepsilon$ of $P_{\varepsilon}(s)$ and $P_{k}(s ; \varepsilon)$ coincide up to the power $\varepsilon^{k}$. The iteration procedure in $[\mathrm{G}, \mathrm{N} 2]$ has been rediscovered in [B2].

A more refined consequence of (1.9) is the following "factorisation theorem." Consider

$$
H_{A}(s ; \varepsilon)=H(s)-B_{\varepsilon}(s),
$$

where

$$
B_{\varepsilon}(s)=-\left(1-2 P_{\varepsilon}(s)\right)\left(i \varepsilon \frac{d}{d s} P_{\varepsilon}(s)-\left[H(s), P_{\varepsilon}(s)\right]\right)
$$

and

$$
i \varepsilon \frac{d}{d s} U_{\varepsilon}^{A}\left(s, s_{0}\right)=H_{A}(s ; \varepsilon) U_{\varepsilon}^{A}\left(s, s_{0}\right) ; \quad U_{\varepsilon}^{A}\left(s_{0}, s_{0}\right)=1
$$

Notice that

$$
\begin{aligned}
H_{A}(s ; \varepsilon)= & P_{\varepsilon}(s) H(s) P_{\varepsilon}(s)+Q_{\varepsilon}(s) H(s) Q_{\varepsilon}(s) \\
& -i \varepsilon\left(P_{\varepsilon}(s) \frac{d}{d s} P_{\varepsilon}(s)+Q_{\varepsilon}(s) \frac{d}{d s} Q_{\varepsilon}(s)\right),
\end{aligned}
$$

where $Q_{\varepsilon}(s)=1-P_{\varepsilon}(s)$. The point is that (without any error!)

$$
P_{\varepsilon}(s)=U_{\varepsilon}^{A}\left(s, s_{0}\right) P_{\varepsilon}\left(s_{0}\right) U_{\varepsilon}^{A}\left(s, s_{0}\right)^{-1}
$$

i.e. $U_{\varepsilon}^{A}\left(s, s_{0}\right)$ is an intertwining operator for $P_{\varepsilon}(s)$. On the other hand if $\Omega_{\varepsilon}\left(s, s_{0}\right)$ is defined by

$$
U_{\varepsilon}\left(s, s_{0}\right)=U_{\varepsilon}^{A}\left(s, s_{0}\right) \Omega_{\varepsilon}\left(s, s_{0}\right)
$$

then

$$
i \varepsilon \frac{d}{d s} \Omega_{\varepsilon}\left(s, s_{0}\right)=U_{\varepsilon}^{A}\left(s, s_{0}\right)^{-1} B_{\varepsilon}(s) U_{\varepsilon}^{A}\left(s, s_{0}\right) \Omega_{\varepsilon}\left(s, s_{0}\right)
$$

which leads (use the fact that $B_{\varepsilon}(s)$ is self-adjoint and then $U_{\varepsilon}^{A}\left(s, s_{0}\right)$ is unitary) to

$$
\left\|\Omega_{\varepsilon}\left(s, s_{0}\right)-1\right\| \leq \varepsilon^{-1} \int_{s_{0}}^{s}\left\|B_{\varepsilon}(u)\right\| d u \leq \varepsilon^{-1} \int_{s_{0}}^{s} \delta(u ; \varepsilon) d u,
$$

i.e. $\Omega_{\varepsilon}\left(s, s_{0}\right)$ is close to the identity. 
The evolution $U_{\varepsilon}^{A}\left(s, s_{0}\right)$ can be further factorised. Let $A_{\varepsilon}\left(s, s_{0}\right)$ be the parallel transport of $P_{\varepsilon}(s)$,

$$
i \frac{d}{d s} A_{\varepsilon}\left(s, s_{0}\right)=\left[i\left(1-2 P_{\varepsilon}(s)\right) \frac{d}{d s} P_{\varepsilon}(s)\right] A_{\varepsilon}\left(s, s_{0}\right) ; \quad A_{\varepsilon}\left(s_{0}, s_{0}\right)=1 .
$$

As well known $[\mathrm{M}, \mathrm{K}, \mathrm{Kr}] A_{\varepsilon}\left(s, s_{0}\right)$ has also the intertwining property

$$
P_{\varepsilon}(s)=A_{\varepsilon}\left(s, s_{0}\right) P_{\varepsilon}\left(s_{0}\right) A_{\varepsilon}\left(s, s_{0}\right)^{-1} .
$$

From (1.18) and (1.23) it follows that if $\Phi_{\varepsilon}\left(s, s_{0}\right)$ is defined by

$$
U_{\varepsilon}^{A}\left(s, s_{0}\right)=A_{\varepsilon}\left(s, s_{0}\right) \Phi_{\varepsilon}\left(s, s_{0}\right)
$$

then

$$
\left[\Phi_{\varepsilon}\left(s, s_{0}\right), P_{\varepsilon}\left(s_{0}\right)\right]=0 .
$$

Moreover one can compute the equation of motion for $\Phi_{\varepsilon}\left(s, s_{0}\right)$ :

$$
\begin{aligned}
i \varepsilon \frac{d}{d s} & \Phi_{\varepsilon}\left(s, s_{0}\right) \\
= & \left\{P_{\varepsilon}\left(s_{0}\right) A_{\varepsilon}^{-1}\left(s, s_{0}\right) H_{A}(s ; \varepsilon) A_{\varepsilon}\left(s, s_{0}\right) P_{\varepsilon}\left(s_{0}\right)\right. \\
& \left.+\left(1-P_{\varepsilon}\left(s_{0}\right)\right) A_{\varepsilon}^{-1}\left(s, s_{0}\right) H_{A}(s ; \varepsilon) A_{\varepsilon}\left(s, s_{0}\right)\left(1-P_{\varepsilon}\left(s_{0}\right)\right)\right\} \Phi_{\varepsilon}\left(s, s_{0}\right) .
\end{aligned}
$$

Summarising:

$$
U_{\varepsilon}\left(s, s_{0}\right)=A_{\varepsilon}\left(s, s_{0}\right) \Phi_{\varepsilon}\left(s, s_{0}\right) \Omega_{\varepsilon}\left(s, s_{0}\right)
$$

where

$A_{\varepsilon}\left(s, s_{0}\right)$ is the parallel transport of $P_{\varepsilon}(s)$,

$\Phi_{\varepsilon}\left(s, s_{0}\right)$ is an evolution which is block diagonal with respect to the decomposition $\mathscr{H}=P_{\varepsilon}\left(s_{0}\right) \mathscr{H} \oplus\left(1-P_{\varepsilon}\left(s_{0}\right)\right) \mathscr{H}$, and

$$
\Omega_{\varepsilon}\left(s, s_{0}\right)-1=\mathscr{O}\left(\varepsilon^{-1} \int_{s_{0}}^{s} \delta(u ; \varepsilon) d u\right) .
$$

Alternatively one can write (1.26) as

$$
U_{\varepsilon}\left(s, s_{0}\right)=A_{\varepsilon}\left(s, s_{0}\right) \Phi_{\varepsilon}\left(s, s_{0}\right)+\odot\left(\varepsilon^{-1} \int_{s_{0}}^{s} \delta(u ; \varepsilon) d u\right) .
$$

The factorisation given by (1.26) (see also [N3, NR1]) is the generalisation of the factorisation theorem of Avron, Seiler and Yaffe [ASY] where $P_{\varepsilon}(s)$ is taken to be $P_{0}(s)$.

Consider now the particular case when $\operatorname{dim} P_{0}(s)=1$ and suppose for definiteness that $H(s)$ is at least $\mathscr{C}^{\infty}$. Due to the fact that $\lim _{\varepsilon \rightarrow 0}\left\|P_{\varepsilon}(s)-P_{0}(s)\right\|=0, \operatorname{dim} P_{\varepsilon}(s)=1$. Let $n_{0}(s)$ be a normalised vector in $P_{0}(s) \mathscr{H}$ which is differentiable with respect to $s$, and

$$
n_{\varepsilon}(s)=P_{\varepsilon}(s) n_{0}(s) /\left\|P_{\varepsilon}(s) n_{0}(s)\right\| .
$$

Then using the fact that by the definition of the parallel transport

$$
\left\langle A_{\varepsilon}\left(s, s_{0}\right) n_{\varepsilon}\left(s_{0}\right), \frac{d}{d s} A_{\varepsilon}\left(s, s_{0}\right) n_{\varepsilon}\left(s_{0}\right)\right\rangle=0
$$


and the fact that $H_{A}(s ; \varepsilon)=H(s)+\Theta\left(\varepsilon^{\infty}\right)$ one obtains

$$
\begin{aligned}
U_{\varepsilon}\left(s, s_{0}\right) n_{\varepsilon}\left(s_{0}\right)= & \exp \left(-i \varepsilon^{-1} \int_{s_{0}}^{s}\left\langle n_{\varepsilon}(u), H(u) n_{\varepsilon}(u)\right\rangle d u\right) \\
& \times \exp \left(\int_{s_{0}}^{s}\left\langle n_{\varepsilon}(u), \frac{d}{d v} n_{\varepsilon}(v)\right\rangle d u\right) n_{\varepsilon}(s)\left(1+C\left(\varepsilon^{\infty}\right)\right) \\
\equiv & \exp \left(-i \phi\left(s, s_{0} ; \varepsilon\right) n_{\varepsilon}(s)\left(1+\circlearrowleft\left(\varepsilon^{\infty}\right)\right) .\right.
\end{aligned}
$$

In particular if $H(s)$ is periodic, i.e. $H(s)$ and all its derivatives at $s=0$ and $s=1$ are equal then

$$
\begin{aligned}
U_{\varepsilon}(1,0) n_{\varepsilon}(0)= & \exp \left(-i \varepsilon^{-1} \int_{s_{0}}^{s}\left\langle n_{\varepsilon}(u), H(u) n_{\varepsilon}(u)\right\rangle d u\right) \\
& \times \exp \left(-\int_{s_{0}}^{s}\left\langle n_{\varepsilon}(u), \frac{d}{d u} n_{\varepsilon}(u)\right\rangle d u\right) n_{\varepsilon}(0)\left(1+\mathscr{C}\left(\varepsilon^{\infty}\right)\right) .
\end{aligned}
$$

which gives the Berry phase to arbitrary order. Notice the separation of the phase in (1.29) into its dynamic and geometric parts, respectively. Using the expansion of $P_{\varepsilon}(s)$ one can obtain explicitly the low orders in the expansion of $\phi\left(s, s_{0} ; \varepsilon\right)$ (see [NR2] for details):

$$
\begin{aligned}
\phi\left(s, s_{0} ; \varepsilon\right)= & \varepsilon^{-1} \int_{s_{0}}^{s} \lambda_{0}(u) d u-i \int_{s_{0}}^{s}\left\langle n_{0}(u), \frac{d}{d u} n_{0}(u)\right\rangle d u \\
& -\varepsilon \int_{s_{0}}^{s}\left\langle\frac{d}{d u} n_{0}(u), Q_{0}(u)\right. \\
& \left.\times\left(H(u)-\lambda_{0}(u)\right)^{-1} Q_{0}(u) \frac{d}{d u} n_{0}(u)\right\rangle d u+\mathscr{C}\left(\varepsilon^{2}\right),
\end{aligned}
$$

where $\lambda_{0}(s)$ is the eigenvalue of $H(s)$ corresponding to $P_{0}(s)$.

For related (heuristic) results in the case when the underlying Hilbert space is two dimensional see [B3].

\section{The Local Theory}

This section contains the construction of $P_{\varepsilon}(s)$ and the proof of the basic estimate (1.9). This part of the theory does not involve differential equations and can be carried in a very general context; however, all the applications are either in the unitary case (i.e. $\mathscr{H}$ is a Hilbert space and $H(s)$ is self-adjoint) or in the finite dimensional case which covers in particular the theory of adiabatic invariants for linear Hamiltonian systems $([\mathrm{BN}]$ and references therein).

Let $s \in(a, b) \subset \mathbf{R}, \mathscr{H}$ be a Banach space, $H(s)$ a family of densely defined closed operators with nonvoid resolvent set. The first main assumption is so called "gap condition": 
G. For all $s \in(a, b), H(s)$ has an isolated bounded part of the spectrum, i.e.

$$
\begin{gathered}
\sigma(H(s))=\sigma_{0}(s) \cup \sigma_{1}(s), \\
\operatorname{dist}\left(\sigma_{0}(s), \sigma_{1}(s)\right)=d(s) \geq d>0, \\
\operatorname{diam} \sigma_{0}(s) \equiv \sup _{z, w \in \sigma_{0}(s)}|z-w|=D(s) \leq D<\infty .
\end{gathered}
$$

The second main assumption concerns the smoothness of $H(s)$ :

S. Let $s \in(a, b)$ and let $\Gamma(s)$ be the contour enclosing $\sigma_{0}(s)$, whose points are at the distance $d(s) / 2$ from $\sigma_{0}(s)$. Suppose there exists a neighbourhood $\mathscr{W}$ of $s$ such that $\Gamma(s) \subset \varrho(H(u))$ for $u \in \mathscr{U}$ and

$\mathbf{S}^{\mathbf{n}} ; n=1,2, \ldots$

$$
\sup _{z \in \Gamma(s)}\left\|\left.\frac{d^{k}}{d u^{k}} R(u ; z)\right|_{u=s}\right\| \equiv r_{k}(s)<\infty, \quad k=0,1, \ldots, n ;
$$

or

$$
\mathbf{S}_{\alpha} ; \alpha \in[0, \infty)
$$

$$
\begin{gathered}
\sup _{z \in \Gamma(s)}\|R(s ; z)\| \leq r(s) ; \\
\sup _{z \in \Gamma(s)}\left\|\left.\frac{d^{k}}{d u^{k}} R(u ; z)\right|_{u=s}\right\| \leq b(s) c(s)^{k}(k !)^{1+\alpha} ; \quad k=1,2, \ldots \\
0 \leq b(s) \leq 1, \quad 0 \leq r(s), c(s)<\infty,
\end{gathered}
$$

where

$$
R(s ; z)=(H(s)-z)^{-1} .
$$

Remarks. 1. Obviously $\mathbf{S}_{\alpha}, \alpha \in[0, \infty)$ implies $\mathbf{S}^{\mathbf{n}}$ for all $n$. The case $\alpha=0$ corresponds to the case when $R(s ; z)$ is holomorphic in a neighbourhood of the real axis, and $\alpha>0$ correspond to Gevrey clases which "interpolate" between the $\mathscr{C}^{\infty}$ case and the holomorphic case.

2. The assumptions in [JP1] imply $\mathbf{G}, \mathbf{S}_{\mathbf{0}}$ and moreover

$$
\int_{-\infty}^{\infty} b(u) d u<\infty
$$

In what follows we shall use the standard notation for the derivatives, e.g.

$$
E_{j}^{(k)}(s)=\frac{d^{k}}{d s^{k}} E_{j}(s) .
$$

Lemma 1. Suppose $\mathbf{G}$ and $\mathbf{S}^{\infty}$ hold true,

$$
P_{0}(s)=(2 \pi i)^{-1} \oint_{\Gamma(s)} R(s ; z) d z
$$

and $E_{j}(s)$ be given by the following recurrent relations:

$$
\begin{gathered}
E_{0}(s)=P_{0}(s) \\
E_{j}(s)=(2 \pi)^{-1} \oint_{\Gamma(s)} R(s ; z)\left\{Q_{0}(s) E_{j-1}^{(1)}(s) P_{0}(s)-P_{0}(s) E_{j-1}^{(1)}(s) Q_{0}(s)\right\} \\
\times R(s ; z) d z+S_{\jmath}(s)-2 P_{0}(s) S_{\jmath}(s) P_{0}(s),
\end{gathered}
$$


where

$$
\begin{gathered}
Q_{0}(s)=1-P_{0}(s) \\
S_{j}(s)=\sum_{m=1}^{j-1} E_{m}(s) E_{j-m}(s) .
\end{gathered}
$$

Then $E_{\jmath}(s), j=0,1, \ldots$, are the unique solution of $(2.2)$,

$$
E_{j}(s)=\sum_{m=0}^{\jmath} E_{m}(s) E_{j-m}(s),
$$

and

$$
i E_{j-1}^{(1)}(s)=\left[H(s), E_{j}(s)\right] .
$$

Warning: As in (2.7) we shall encounter many times expressions like

$$
[H, L]=H L-L H,
$$

where $H$ is a densely defined unbounded operator and $L$ is bounded. In all the cases below the situation is as follows: $L \mathscr{D} \subset \mathscr{Q}$ (= the domain of $H$ ) so that $H L-L H$ is well defined on $\mathscr{D}$; moreover

$$
\|(H L-L H) f\| \leq M\|f\|, \quad M<\infty, \quad f \in \mathscr{D} .
$$

We shall always understand $[H, L]$ as the extension by continuity of $H L-L H$ defined on $\mathscr{D}$.

Proof. Since during the proof below $s$ is kept fixed we shall usually omit it. The proof is by induction. Notice that $E_{m} \mathscr{H} \subset \mathscr{D}(H)$ for $m=0,1, \ldots, j-1$ imply $E_{3} \mathscr{H} \subset \mathscr{Z}(H)$. Compute (use (2.5), $[A, B C]=B[A, C]+[A, B] C,(2.6)$ and (2.7))

$$
\begin{aligned}
{\left[H, S_{j}\right] } & =\sum_{m=1}^{\jmath-1}\left[H, E_{m} E_{\jmath-m}\right]=i \sum_{m=1}^{\jmath-1}\left(E_{m} E_{j-m-1}^{(1)}+E_{m-1}^{(1)} E_{\jmath-m}\right) \\
& =i\left(E_{\jmath-1}^{(1)}-E_{0} E_{j-1}^{(1)}-E_{\jmath-1}^{(1)} E_{0}\right)=-i\left(P_{0} E_{j-1}^{(1)} P_{0}-Q_{0} E_{j-1}^{(1)} Q_{0}\right)
\end{aligned}
$$

whereof

$$
\begin{gathered}
{\left[H, P_{0} S_{j} P_{0}\right]=-i P_{0} E_{j-1}^{(1)} P_{0},} \\
{\left[H, Q_{0} S_{j} Q_{0}\right]=i Q_{0} E_{j-1}^{(1)} Q_{0},} \\
{\left[H, P_{0} S_{j} Q_{0}\right]=\left[H, Q_{0} S_{j} P_{0}\right]=0 .}
\end{gathered}
$$

Compute (use (2.3) and (2.8)).

$$
P_{0}\left[H, E_{j}\right] P_{0}=i P_{0} E_{\jmath-1}^{(1)} P_{0},
$$

Analogously

$$
Q_{0}\left[H, E_{j}\right] Q_{0}=i Q_{0} E_{\jmath-1}^{(1)} Q_{0} .
$$

From (2.3), (2.10), (2.1) and the identity $H R(z)=1+z R(z)$,

$$
P_{0}\left[H, E_{j}\right] Q_{0}=-\left[H,(2 \pi)^{-1} \oint_{\Gamma} R(z) P_{0} E_{j-1}^{(1)} Q_{0} R(z) d z\right]=i P_{0} E_{\jmath-1}^{(1)} Q_{0}
$$


and

$$
Q_{0}\left[H, E_{j}\right] P_{0}=i P_{0} E_{\jmath-1}^{(1)} Q_{0} .
$$

Now (2.11-14) imply (2.7) for $j$. Further, consider (2.6) written in the form

$$
E_{j}=P_{0} E_{\jmath}+E_{j} P_{0}+S_{j}
$$

To verify (2.15) amounts to see that

$$
\begin{gathered}
P_{0} E_{j} P_{0}=-P_{0} S_{\jmath} P_{0}, \\
Q_{0} E_{\jmath} Q_{0}=Q_{0} S_{j} Q_{0}, \\
Q_{0} S_{j} P_{0}=P_{0} S_{j} Q_{0}=0 .
\end{gathered}
$$

Now (2.16), (2.17) follow from (2.3) while (2.18) follows from (2.10) and the following simple fact:

Proposition 1. Let $T$ be a bounded operator. If

$$
\left[H, P_{0} T Q_{0}\right]=0
$$

then

$$
P_{0} T Q_{0}=0
$$

Proof. Let $\psi \in \mathscr{D}(H), z \in \varrho(H)$. If

$$
H P_{0} T Q_{0} \psi=P_{0} T Q_{0} H \psi,
$$

then

$$
(H-z) P_{0} T Q_{0} \psi=P_{0} T Q_{0}(H-z) \psi
$$

whereof for $\psi=(H-z)^{-1} g, g \in \mathscr{H}$,

$$
(H-z)^{-1} P_{0} T Q_{0} g=P_{0} T Q_{0}(H-z)^{-1} g .
$$

Integrate the above relation over $\Gamma$, use (2.1) and remind that

$$
P_{0}^{2}=P_{0}, \quad Q_{0} P_{0}=0 \text {. }
$$

Consider now the uniqueness of $E_{\jmath}$. Suppose uniqueness proved for $E_{1}, \ldots, E_{j-1}$; $E_{\jmath}, \hat{E}_{j}$ satisfy (2.6) and (2.7). Then from (2.7)

$$
\left[H, P_{0}\left(E_{j}-\hat{E}_{j}\right) Q_{0}\right]=\left[H, Q_{0}\left(E_{\jmath}-\hat{E}_{\jmath}\right) P_{0}\right]=0
$$

and the use of Proposition 1 gives

$$
P_{0}\left(E_{j}-\hat{E}_{\jmath}\right) Q_{0}=Q_{0}\left(E_{\jmath}-\hat{E}_{j}\right) P_{0}=0 .
$$

On the other hand from (2.6) (see (2.15))

$$
P_{0}\left(E_{j}-\hat{E}_{j}\right) P_{0}=Q_{0}\left(E_{j}-\hat{E}_{\jmath}\right) Q_{0}=0,
$$

and the proof of Lemma 1 is finished.

Consider now the problem of estimating $\left\|E_{j}(s)\right\|,\left\|E_{j}^{(1)}(s)\right\|$. The following lemma is obvious

Lemma 2. Suppose $\mathbf{G}, \mathbf{S}^{\infty}$ hold true. Then there exist $e_{j}(s), f_{j}(s)<\infty$ (computable from $r_{k}(s)$ and the length of $\left.\Gamma(s)\right)$ such that for $j=0,1, \ldots$,

$$
\left\|E_{\jmath}(s)\right\| \leq e_{j}(s), \quad\left\|E_{j}^{(1)}(s)\right\| \leq f_{j}(s) .
$$


The main technical point is to control $\left\|E_{j}(s)\right\|,\left\|E_{j}^{(1)}(s)\right\|$ as $j \rightarrow \infty$ when $\mathbf{G}, \mathbf{S}_{\alpha}$ hold true.

Lemma 3. Suppose $\mathbf{G}, \mathbf{S}_{\alpha}$ hold true. Then there exist $A(s), a(s)<\infty A(s) \geq 1$ such that for $j \geq 1$,

$$
\left\|E_{\jmath}^{(k)}(s)\right\| \leq b(s) A(s)^{j-2} a(s)^{j+k} \frac{[(j+k) !]^{1+\alpha}}{(k+1)^{2}(j+1)^{2}} .
$$

Proof. We shall prove by induction that (2.19) "propagates" through the recurrence. We shall use repeatedly the following simple fact:

Proposition 2. Let $M(s), N(s) \in \mathscr{C} \infty$ be operator valued functions, $p, q=0,1, \ldots$ Suppose

$$
\begin{gathered}
\left\|M^{(k)}(s)\right\| \leq m(s) a(s)^{p+k} \frac{[(p+k) !]^{1+\alpha}}{(k+1)^{2}} \\
\left\|N^{(k)}(s)\right\| \leq n(s) a(s)^{q+k} \frac{[(q+k) !]^{1+\alpha}}{(k+1)^{2}}
\end{gathered}
$$

Then

$$
\left\|(M(s) N(s))^{(k)}\right\| \leq f m(s) n(s) a(s)^{p+q+k} \frac{[(p+q+k) !]^{1+\alpha}}{(k+1)^{2}},
$$

where $f$ is the (absolute) constant appearing in the inequality

$$
\sum_{l=0}^{k} \frac{1}{(l+1)^{2}(k+1-l)^{2}} \leq f \frac{1}{(k+1)^{2}} .
$$

Proof of Proposition 2. By (2.20) and the Leibnitz formula (omit $s$ and use the standard notation $\left.C_{l}^{k}=\frac{k !}{l !(k-l) !}\right)$

$$
\begin{aligned}
\left\|(M N)^{(k)}\right\| \leq & \sum_{l=0}^{k} \frac{k !}{l !(k-l) !}\left\|M^{(l)}\right\|\left\|N^{(k-l)}\right\| \leq m n a^{p+q+k}[(p+q+k) !]^{1+\alpha} \\
& \times \sum_{l=0}^{k} \frac{C_{k}^{l}}{\left(C_{p+q+k}^{p+l}\right)^{1+\alpha}} \frac{1}{(l+1)^{2}(k+1-l)^{2}} .
\end{aligned}
$$

Now

$$
\begin{gathered}
\left(C_{p+q+k}^{p+l}\right)^{1+\alpha} \geq C_{p+q+k}^{p+l} \geq C_{k}^{l}, \\
\sum_{l=0}^{k} \frac{1}{(l+1)^{2}(l+1-l)^{2}}=\left(\sum_{l=0}^{[k / 2]}+\sum_{l=[k / 2]+1}^{k}\right) \frac{1}{(l+1)^{2}(k+1-l)^{2}} \\
\leq 2 \frac{1}{([k / 2]+1)^{2}} \sum_{l=0}^{\infty} \frac{1}{(l+1)^{2}} \leq f \frac{1}{(k+1)^{2}}
\end{gathered}
$$

Insert (2.23) and (2.24) into (2.22) to finish the proof of Proposition 2. 
We shall use the following obvious consequence of $\mathbf{S}_{\alpha}$ : for arbitrary $K(s)<\infty$ there exists $a(s)<\infty$ such that (see $\mathbf{S}^{\mathbf{n}}$ for the definition of $r_{k}(s)$ )

$$
\begin{gathered}
r_{k}(s) \leq a(s)^{k}(k !)^{1+\alpha} \frac{1}{(k+1)^{2}}, \quad k=0,1, \ldots, \\
r_{k}(s) \leq \frac{b(s)}{K(s)} a(s)^{k}(k !)^{1+\alpha} \frac{1}{(k+1)^{2}}, \quad k=1,2, \ldots
\end{gathered}
$$

Notice that from (2.1) and (2.4),

$$
\left\|P_{0}(s)^{k}\right\|=\left\|Q_{0}(s)^{(k)}\right\| \leq \frac{L(s)}{2 \pi} r_{k}(s),
$$

where $L(s)$ is the length of $\Gamma(s)$.

Consider first $j=1$ (again $s$ is omitted). Since by definition $S_{1}=0$,

$$
E_{1}=(2 \pi)^{-1} \oint_{\Gamma} R(z)\left(Q_{0} P_{0}^{(1)} P_{0}-P_{0} P_{0}^{(1)} Q_{0}\right) R(z) d z .
$$

Since $\left(P_{0}^{(1)}\right)^{(k)}=P_{0}^{(k+1)}$ from (2.25) and (2.26):

$$
\left\|\left(P_{0}^{(1)}\right)^{(k)}\right\| \leq \frac{L}{2 \pi} \frac{b}{K} a^{k+1}[(k+1) !]^{1+\alpha} \frac{1}{(k+1)^{2}} .
$$

Using Proposition 2, (2.25), (2.27), and (2.29) one obtains from (2.28),

$$
\left\|E_{1}^{(k)}\right\| \leq A^{-1} b a^{k+1}[(k+1) !]^{1+\alpha} \frac{1}{4(k+1)^{2}}
$$

with

$$
A^{-1}=(1 / 2)(8 L / \pi)^{4} / K
$$

i.e. (2.19) for $j=1$. Notice that we can choose $A$ as large as we want.

Suppose now (2.19) holds true for $m=1,2, \ldots, j-1$ and take $E_{j}$ as given by (2.3). Consider first $S_{j}$. Using Proposition 2 and (2.21),

$$
\left\|S_{\jmath}^{(k)}\right\| \leq f^{2} b^{2} A^{j-4} a^{k+\jmath}[(k+j) !]^{1+\alpha} \frac{1}{(k+1)^{2}(j+1)^{2}} .
$$

Using again twice Proposition 2, (2.32), (2.27)

$$
\left\|\left(P_{0} S_{j} P_{0}\right)^{(k)}\right\| \leq(L / 2 \pi)^{2} f^{4} b^{2} A^{j-4} a^{k+\jmath}[(k+j) !]^{1+\alpha} \frac{1}{(k+1)^{2}(j+1)^{2}} .
$$

Consider now the integral term in (2.3). From the induction hypothesis one has for $j \geq 2$

$$
\left\|\left(E_{\jmath-1}^{(1)}\right)^{(k)}\right\| \leq 3 b A^{j-3} a^{k+j}[(k+j) !]^{1+\alpha} \frac{1}{(k+1)^{2}(j+1)^{2}} .
$$

Applying again four times Proposition 2, (2.25) and (2.27) one obtains

$$
\begin{aligned}
& \left\|\left\{(2 \pi)^{-1} \oint_{\Gamma} R(z)\left[Q_{0} E_{j-1}^{(1)} P_{0}-P_{0} E_{j-1}^{(1)} Q_{0}\right] R(z) d z\right\}^{(k)}\right\| \\
& \quad \leq 6(L / 2 \pi)^{3} f^{4} b A^{\jmath-3} a^{k+j}[(k+j) !]^{1+\alpha} \frac{1}{(k+1)^{2}(j+1)^{2}} .
\end{aligned}
$$


Combining (2.32), (2.33), and (2.35) one obtains from (2.3):

$$
\begin{aligned}
\left\|E_{j}^{(k)}\right\| \leq & \left\{6(L / 2 \pi)^{3} f^{4} A^{-1}+f^{2} b A^{-2}+f^{4}(L / 2 \pi)^{2} b A^{-2}\right\} \\
& \times b A^{j-2} a^{k+\jmath}[(k+j) !]^{1+\alpha} \frac{1}{(k+1)^{2}(j+1)^{2}} .
\end{aligned}
$$

Since $A$ can be made arbitrarily large (see the remark following (2.31)) the expression in the curly bracket can be made smaller than one and the proof of Lemma 1 is finished.

In what follows we shall use Lemma 3 in the following form:

Lemma 4. Suppose $\mathbf{G}, \mathbf{S}_{\alpha}$ hold true. Then there exists $g(s)<\infty$ (depending upon $L(s), r(s), c(s))$ such that

$$
\begin{gathered}
\left\|E_{j}(s)\right\| \leq b(s) g(s)^{j}(j !)^{1+\alpha} \\
\left\|E_{j}^{(1)}(s)\right\| \leq b(s) g(s)^{j+1}[(j+1) !]^{1+\alpha} .
\end{gathered}
$$

Proof. Take $g(s)=A(s) a(s)$ and recall that $A(s) \geq 1$.

We need a few elementary facts about projections. Let $P$ be a bounded idempotent. Then (see e.g. [K, Chap. III.4]) $\mathscr{H}=\mathscr{H} l \mathscr{N}^{\circ}$ such that if $f=m+n, m \in \mathscr{M l}$, $n \in \mathscr{N}$ then $P f=m$, i.e. $P$ is a projection. Recall that if $P$ is a bounded projection then $\sigma(P)=\{0,1\}$. Let now $T$ be bounded and "almost" idempotent. The problem is whether $T$ is close to an idempotent.

Proposition 3. Let $T_{\delta}, \delta \rightarrow 0$ be a family of bounded operators such that:

i) There exists a bounded projection $P$ such that

$$
\lim _{\delta \rightarrow 0}\left\|T_{\delta}-P\right\|=0
$$

ii)

$$
\left\|T_{\delta}^{2}-T_{\delta}\right\| \leq \delta
$$

Then for sufficiently small $\delta,\{|z-1|=1 / 2\} \subset \varrho\left(T_{\delta}\right)$ and there exists $k<\infty$ such that

$$
\left\|T_{\delta}-\frac{1}{2 \pi i} \oint_{|z-1|=1 / 2}\left(T_{\delta}-z\right)^{-1} d z\right\| \leq k \delta .
$$

Proof. By perturbation theory from (2.39) it follows that for sufficiently small $\delta$ the spectrum of $T_{\delta}$ is concentrated near 0 and 1 so that

$$
P_{\delta}=\frac{1}{2 \pi i} \oint_{|z-1|=1 / 2}\left(T_{\delta}-z\right)^{-1} d z
$$

is well defined, and moreover

$$
1-P_{\delta}=\frac{1}{2 \pi i} \oint_{|z|=1 / 2}\left(T_{\delta}-z\right)^{-1} d z .
$$


By direct computation

$$
\begin{aligned}
T_{\delta}-P_{\delta} & =\frac{1}{2 \pi i} \oint_{|z-1|=1 / 2}\left[T_{\delta}(1-z)^{-1}-\left(T_{\delta}-z\right)^{-1}\right] d z \\
& =\left(T_{\delta}-1\right) P_{\delta}+\left(T_{\delta}^{2}-T_{\delta}\right) A_{\delta}
\end{aligned}
$$

where

$$
A_{\delta}=\frac{1}{2 \pi i} \oint_{|z-1|=1 / 2}\left(T_{\delta}-z\right)^{-1}(1-z)^{-1} d z
$$

so that

$$
T_{\delta}\left(1-P_{\delta}\right)=\left(T_{\delta}^{2}-T_{\delta}\right) A_{\delta}
$$

Repeating the computation for $\tilde{T}_{\delta}=1-T_{\delta}$ and noticing that

$$
T_{\delta}^{2}-T_{\delta}=\tilde{T}_{\delta}^{2}-\tilde{T}_{\delta}, \quad \tilde{P}_{\delta}=1-P_{\delta}
$$

one obtains

$$
\left(1-T_{\delta}\right) P_{\delta}=\left(T_{\delta}^{2}-T_{\delta}\right) \tilde{A}_{\delta}
$$

Subtracting (2.44) from (2.43) one obtains

$$
T_{\delta}-P_{\delta}=\left(T_{\delta}^{2}-T_{\delta}\right)\left(A_{\delta}-\tilde{A}_{\delta}\right)
$$

and the proof is finished.

Remark. In the self-adjoint case i) is not needed and the proof is an easy consequence of the functional calculus. In fact we suspect that i) can be replaced by the condition that $\left\|T_{\delta}\right\|$ is uniformly bounded as $\delta \rightarrow 0$.

Consider now

$$
T_{N}(s ; \varepsilon)=\sum_{j=0}^{N} E_{j}(s) \varepsilon^{j} .
$$

From (2.7)

$$
i \varepsilon T_{N}^{(1)}(s ; \varepsilon)-\left[H(s), T_{N}(s ; \varepsilon)\right]=i \varepsilon^{N+1} E_{N}^{(1)}(s)
$$

Let

$$
N_{\varepsilon}+1=\left[\left(\frac{1}{g(s) \varepsilon}\right)^{1 / 1+\alpha}\right]
$$

where $[. .$.$] means the integer part. Define$

$$
T_{\varepsilon}(s)= \begin{cases}T_{n-1}(s ; \varepsilon) & \text { if } \mathbf{S}^{\mathbf{n}} \text { holds true } \\ T_{N_{\varepsilon}}(s ; \varepsilon) & \text { if } \mathbf{S}_{\alpha} \text { holds true. }\end{cases}
$$

We shall consider explicitly only the $\mathbf{S}_{\alpha}$ case; the $\mathbf{S}^{\mathbf{n}}$ case is simpler and we shall only state the result (see Theorem 1 below).

Lemma 5. i)

$$
\lim _{\varepsilon \rightarrow 0}\left\|T_{\varepsilon}(s)-P_{0}(s)\right\|=0 .
$$

ii) There exist (absolute) constants $c_{1}, c_{2}$ such that

$$
\left\|T_{\varepsilon}^{2}(s)-T_{\varepsilon}(s)\right\| \leq c_{1} b(s) \exp \left[-c_{2} /(\varepsilon g(s))^{1 / 1+\alpha}\right] .
$$


Proof. Denote $\eta=\varepsilon g$ (variable $s$ omitted). From Lemma 4:

$$
\left\|T_{\varepsilon}-P_{0}\right\| \leq b \eta \sum_{j=1}^{N_{\varepsilon}} \eta^{j-1}(j !)^{1+\alpha} .
$$

From the Stirling formula

$$
j !=\exp [j(\ln j-1)] \sqrt{2 \pi j}(1+\mathscr{O}(1 / j))
$$

and the fact that (see (2.47)) for all $j \leq N_{\varepsilon}+1, \ln \eta+(1+\alpha) \ln j \leq 0$ one obtains

$$
\eta^{\jmath-1}(j !)^{1+\alpha} \leq \text { const } \exp [-(1+\alpha) j] j^{3(1+\alpha) / 2}
$$

so that

$$
\left\|T_{\varepsilon}-P_{0}\right\| \leq \text { const } b \eta \sum_{j=1}^{\infty} \exp [-(1+\alpha) j] j^{3(1+\alpha) / 2}
$$

which proves (2.49).

From (2.6)

$$
\left\|T_{\varepsilon}^{2}-T_{\varepsilon}\right\| \leq \sum_{l=N_{\varepsilon}+1}^{2 N_{\varepsilon}} \varepsilon^{l}\left\|_{\jmath+m=l ; 0 \leq \jmath, m \leq N_{\varepsilon}} E_{\jmath} E_{m}\right\| .
$$

From Lemma 4 (recall that $b \leq 1$ )

$$
\left\|\sum_{j+m=l ; 0 \leq j, m \leq N_{\varepsilon}} E_{j} E_{m}\right\| \leq \sum_{j=0}^{l}\left\|E_{j}\right\|\left\|E_{l-j}\right\| \leq b(l+1) g^{l}(l !)^{1+\alpha} .
$$

Inserting (2.52) into (2.51), using again Stirling formula, the fact that for $l \leq 2 N_{\varepsilon}$, $\ln \eta+(1+\alpha) \ln l \leq(1+\alpha) \ln 2$ and the fact that for all $q, \beta>0, \sup j^{q} \exp (-\beta \bar{j})<\infty$ one obtains (2.50).

We shall define now $P_{\varepsilon}(s)$ by

$$
P_{\varepsilon}(s)=\frac{1}{2 \pi i} \oint_{|z-1|=1 / 2}\left(T_{\varepsilon}(s)-z\right)^{-1} d z .
$$

The definition makes sense for sufficiently small $\varepsilon$, due to Lemma 5 and Proposition 3. Using the identity

$$
(T-z)^{-1}=z^{-1}\left(T(T-z)^{-1}-1\right)
$$

and the fact that $T_{\varepsilon}(s) \mathscr{H} \subset \mathscr{D}(H(s))$ (see the proof of Lemma 1) one concludes that $P_{\varepsilon}(s) \not \mathscr{C} \subset \mathscr{D}(H(s))$.

In the theorem below "for sufficiently small $\varepsilon$ " is a shorthand for "there exists $\varepsilon_{0}>0$ such that for $0 \leq \varepsilon \leq \varepsilon_{0}$."

Theorem 1. a) Suppose $\mathbf{G}, \mathbf{S}^{\mathbf{n}}$ hold true. Then:

ai)

$$
P_{\varepsilon}(s)=\sum_{j=0}^{n-1} E_{\jmath}(s) \varepsilon^{\jmath}+\varepsilon^{n} R(s ; \varepsilon)
$$

with $\|R(s ; \varepsilon)\|$ uniformly bounded for sufficiently small $\varepsilon$. 
aii) There exists $q(s)$ (depending upon $L(s), r_{k}(s)$ ) such that for sufficiently small $\varepsilon$ :

$$
\left\|i \varepsilon \frac{d}{d s} P_{\varepsilon}(s)-\left[H(s), P_{\varepsilon}(s)\right]\right\| \leq \varepsilon^{n} q(s) \equiv \delta_{n}(s ; \varepsilon) .
$$

b) Suppose $\mathbf{G}, \mathbf{S}_{\alpha}$ hold true. Then:

bi) In the sense of asymptotic series

$$
P_{\varepsilon}(s)=\sum_{\jmath=0}^{\infty} E_{\jmath}(s) \varepsilon^{\jmath}
$$

bii) There exists constants $k_{1}<\infty$ and $0<k_{2}$ (depending upon $L(s), r(s), c(s)$ ) such that for sufficiently small $\varepsilon$ :

$$
\left\|i \varepsilon \frac{d}{d s} P_{\varepsilon}(s)-\left[H(s), P_{\varepsilon}(s)\right]\right\| \leq k_{1}(s) b(s) \exp \left[-\frac{k_{2}(s)}{\varepsilon^{1 / 1+\alpha}}\right] \equiv \delta_{\alpha}(s ; \varepsilon) .
$$

Remark. Notice that in particular

$$
\lim _{\varepsilon \rightarrow 0}\left\|P_{\varepsilon}(s)-P_{0}(s)\right\|=0 .
$$

Proof of Theorem 1b. The asymptotic expansion (2.56) is a direct consequence of (2.45), (2.48), (2.50), (2.53), and Proposition 3.

Let us compute now (use (2.46))

$$
\begin{aligned}
i \varepsilon P_{\varepsilon}^{(1)}= & \frac{1}{2 \pi i} \oint_{|z-1|=1 / 2}\left(T_{\varepsilon}-z\right)^{-1} i \varepsilon T_{\varepsilon}^{(1)}\left(T_{\varepsilon}-z\right)^{-1} d z \\
= & -\frac{1}{2 \pi i} \oint_{|z-1|=1 / 2}\left(T_{\varepsilon}-z\right)^{-1}\left[H, T_{\varepsilon}\right]\left(T_{\varepsilon}-z\right)^{-1} d z \\
& -\varepsilon^{N_{\varepsilon}+1} \frac{1}{2 \pi} \oint_{|z-1|=1 / 2}\left(T_{\varepsilon}-z\right)^{-1} E_{N_{\varepsilon}}^{(1)}\left(T_{\varepsilon}-z\right)^{-1} d z
\end{aligned}
$$

Now using the identity

$$
-\left(T_{\varepsilon}-z\right)^{-1}\left[H, T_{\varepsilon}\right]\left(T_{\varepsilon}-z\right)^{-1}=\left[H,\left(T_{\varepsilon}-1\right)^{-1}\right]
$$

one obtains

$$
\left\|i \varepsilon P_{\varepsilon}^{(1)}-\left[H, P_{\varepsilon}\right]\right\| \leq \mathrm{const} \varepsilon^{N_{\varepsilon}+1}\left\|E_{N_{\varepsilon}}^{(1)}\right\|
$$

from which (2.57) follows (use Lemma 4 and again the Stirling formula).

\section{Intertwining Evolutions and Factorisation Theorems}

We shall start with some variations on the well known Krein-Kato lemma [K2, Kr]. Let $Q(s)$ be a norm differentiable family of bounded projections, $s \in(a, b) \subset \mathbf{R}$. 
The problem is to find families, $V\left(s, s_{0}\right)$, of bounded with bounded inverse operators intertwining $Q(s)$, i.e.

$$
Q(s)=V\left(s, s_{0}\right) Q\left(s_{0}\right) V^{-1}\left(s, s_{0}\right) .
$$

We shall call $V\left(s, s_{0}\right)$ an intertwining evolution for $Q(s)$. In what follows we shall describe a large class of intertwining evolutions for $Q(s)$ given as solutions of Schrödinger type equations,

$$
i \frac{d}{d s} V\left(s, s_{0}\right)=K(s) V\left(s, s_{0}\right) ; \quad V\left(s_{0}, s_{0}\right)=1
$$

with suitably chosen $K(s)$. If we like to allow unbounded $K(s)$, some conditions are to be met in order (3.2) to have a solution. For simplicity we shall reproduce below some results only for families of orthogonal projections in Hilbert spaces (see e.g. [T] also for the conditions in the general case).

Proposition 4. Suppose $N(s)$ to be a family of self-adjoint operators in a Hilbert space $\mathscr{H}$, satisfying

i) $\mathscr{D}(N(s))=\mathscr{Q}$ independent of $s$,

ii) $N(s)\left(N\left(s_{0}\right)-i\right)^{-1}, s_{0} \in(a, b)$ is strongly differentiable on $(a, b)$.

Then the equation

$$
\frac{d}{d s} W\left(s, s_{0}\right)=N(s) W\left(s, s_{0}\right) ; \quad W\left(s_{0}, s_{0}\right)=1
$$

has a unique unitary strongly continuous solution.

The following is a generalisation of the Krein-Kato lemma:

Lemma 6. Suppose:

i) $Q(s)$ is a norm differentiable family of orthogonal projections in a Hilbert space $\mathscr{H}$.

ii) $N(s)$ satisfy the conditions of Proposition 4.

iii) $Q(s) \mathscr{D} \subset \mathscr{D}, \frac{d}{d s} Q(s) \mathscr{D} \subset \mathscr{Q},\|[N(s), Q(s)]\| \leq M<\infty$.

Then

$$
K_{N}(s)=N(s)+(1-2 Q(s))\left(i \frac{d}{d s} Q(s)-[N(s), Q(s)]\right)
$$

is self-adjoint on $\mathscr{D}$, satisfies the conditions of Proposition 4 and $A_{N}\left(s, s_{0}\right)$ given by

$$
i \frac{d}{d s} A_{N}\left(s, s_{0}\right)=K_{N}(s) A_{N}\left(s, s_{0}\right) ; \quad A_{N}\left(s_{0}, s_{0}\right)=1
$$

is an intertwining evolution for $Q(s)$.

Proof. Using $Q^{(1)} Q+Q Q^{(1)}=Q^{(1)}$ it follows at once that the second term in the r.h.s. of (3.3) is self-adjoint and since it is bounded, by perturbation theory $K_{N}$ is self-adjoint. Moreover due to iii) it satisfies the conditions in Proposition 4. Consider:

$$
f(s)=A_{N}^{-1}\left(s, s_{0}\right) Q(s) A_{N}\left(s, s_{0}\right) .
$$

By direct computation, for $\psi \in \mathscr{D}$,

$$
i f^{(1)} \psi=A_{N}^{-1}\left\{\left[Q, K_{N}\right]+i Q^{(1)}\right\} A_{N}
$$


and using that $Q Q^{(1)} Q=0$ one can verify that the expression in the curly bracket vanishes.

We shall use the following direct consequence of the above lemma.

Lemma 7. Let $\Phi_{N}\left(s, s_{0}\right)$ be defined by

$$
A_{N}\left(s, s_{0}\right)=A_{0}\left(s, s_{0}\right) \Phi_{N}\left(s, s_{0}\right) .
$$

Then

$$
\left[\Phi_{N}\left(s, s_{0}\right), Q\left(s_{0}\right)\right]=0
$$

and for $\psi \in \mathscr{Q}$,

$$
\begin{aligned}
i \frac{d}{d s} \Phi_{N}\left(s, s_{0}\right) \psi= & \left\{Q\left(s_{0}\right) A_{0}^{-1}\left(s, s_{0}\right) N(s) A_{0}\left(s, s_{0}\right) Q\left(s_{0}\right)+\left(1-Q\left(s_{0}\right)\right) A_{0}^{-1}\left(s, s_{0}\right)\right. \\
& \left.\times N(s) A_{0}\left(s, s_{0}\right)\left(1-Q\left(s_{0}\right)\right)\right\} \Phi_{N}\left(s, s_{0}\right) \psi
\end{aligned}
$$

Applying the above scheme, with $\varepsilon^{-1} H(s)$ as $N(s)$, to the family, $P_{\varepsilon}(s)$, constructed in the previous section one obtains:

Theorem 2. Suppose $H(s)$ satisfies $\mathbf{G}, \mathbf{S}$, and either $\operatorname{dim} \mathscr{H}<\infty$ or $H(s)$ satisfies also the conditions in Proposition 4. Let $P_{\varepsilon}(s)$ as given by the construction in Sect.2, and $U_{\varepsilon}\left(s, s_{0}\right), B_{\varepsilon}(s), U_{\varepsilon}^{A}\left(s, s_{0}\right), A_{\varepsilon}\left(s, s_{0}\right), \Phi_{\varepsilon}\left(s, s_{0}\right), \Omega_{\varepsilon}\left(s, s_{0}\right)$ be given by (1.1), (1.16), (1.17), (1.22), (1.24), and (1.19) respectively. Then (1.18), (1.20), (1.23), and (1.25) are valid. If in addition $H(s)$ is self-adjoint then (1.21), where $\delta(s ; \varepsilon)$ is given by (2.55) or (2.57), holds true.

\section{Extensions}

Some applications [N4] require some straigthforward extensions of the above theory and we shall mention a few.

a) The generator, $H=H(s ; \lambda)$ depends on an additional parameter $\lambda \in \Lambda$, where $\lambda$ is a topological space. Suppose that $H(s ; \lambda)$ satisfies $\mathbf{G}, \mathbf{S}$ uniformly on $\Lambda$. Moreover suppose $(H(s ; \lambda)-z)^{-1}$ is norm differentiable with respect to $\lambda$ and its derivative satisfies $\mathbf{S}$ uniformly in $\lambda$. Then $P_{\varepsilon}(s ; \lambda)$ is norm differentiable with respect to $\lambda$ and its derivative has an asymptotic expansion in $\varepsilon$ which can be obtained by term by term differentiation of the asymptotic expansion of $P_{\varepsilon}(s ; \lambda)$.

b) The generator $H=H(s ; \varepsilon)$ depends itself on $\varepsilon$. The theory applies without any changes as far as $H=H(s ; \varepsilon)$ satisfies $\mathbf{G}, \mathbf{S}$ uniformly for $\varepsilon \in\left[0, \varepsilon_{0}\right], \varepsilon_{0}>0$. Of course in this case $E_{j}$ will depend also on $\varepsilon$; if $P_{0}(s ; \varepsilon)$ has a series expansion in $\varepsilon$ then the same is true for $E_{j}(s ; \varepsilon)$.

Finally, let us stress that Theorem 1 implies the bound (1.21) for $\Omega$ only in the self-adjoint case. In the general case in order to obtain bounds on $\Omega-1$ it is necessary to control $\left\|U_{\varepsilon}^{A}\left(s, s_{0}\right)\right\|$ (see [BN, NR] for specific problems in which this has been done). As far as the needed bounds are obtained all the theory can be applied; in particular $(1.28-30)$ remain valid, but in this case $\phi\left(s, s_{0} ; \varepsilon\right)$ becomes complex. 


\section{References}

[AHS] Avron, J.E., Howland, J.S., Simon, B.: Adiabatic theorems for dense point spectra. Commun. Math. Phys. 128, 497-507 (1990)

[ASY] Avron, J.E., Seiler, R., Yaffe, L.G.: Adiabatic theorem and applications to the quantum Hall effect. Commun. Math. Phys. 110, 33-49 (1987)

[BF] Born, M., Fock, V.: Beweis des Adiabatensatzes. Z. Phys. 5, 165-180 (1928)

[BN] Boutet de Monvel, A., Nenciu, G.: On the theory of adiabatic invariants for linear Hamiltonian systems. C.R. Acad. Sci. Paris 310, 807-810 (1990)

[B1] Berry, M.V.: Geometric amplitude factors in adiabatic quantum transitions. Proc. R. Soc. Lond. A 430, 405-411 (1990)

[B2] Berry, M.V.: Quantum phase corrections from adiabatic iteration. Proc. R. Soc. Lond. A 414, 31-46 (1987)

[B3] Berry, M.V.: Histories of adiabatic quantum transitions. Proc. R. Soc. Lond. A 429, 61-72 (1990)

[G] Garrido, L.M.: Generalised adiabatic invariance. J. Math. Phys. 5, 335-362 (1964)

[H1] Hagedorn, G.: Proof of the Landau-Zener formula in an adiabatic limit with small eigenvalue gaps. Commun. Math. Phys. 136, 433-449 (1991)

[H2] Hagedorn, G.: Adiabatic expansions near eigenvalue crossing. Ann. Phys. 196, 278-296 (1989)

[HK] Hu, J., Kruskal, M.: Reflection coefficient beyond all orders for singular problems. I, II. J. Math. Phys. 32, 2400-2405 (1991); 32, 2676-2678 (1991)

[JKP] Joye, A., Kunz, H., Pfister, C-E.: Exponential decay and geometric aspect of transition probabilities in the adiabatic limit. Ann. Phys. 208, 299-332 (1991)

[JMP] Joye, A., Mileti, G., Pfister, C-E.: Interferences in adiabatic transition probabilities mediated by Stokes lines. Phys. Rev. A 44, 4280-4295 (1991)

[JP1] Joye, A., Pfister, C-E.: Exponentially small adiabatic invariant for the Schrödinger equation. Commun. Math. Phys. 140, 15-41 (1991)

[JP2] Joye, A., Pfister, C-E.: Full asymptotic expansion of transition probabilities in the adiabatic limit. J. Phys. A: Math. Gen. 24, 753-766 (1991)

[K1] Kato, T.: On the adiabatic theorem of quantum mechanics. J. Phys. Soc. Japan 5, 435-439 (1950)

[K2] Kato, T.: Perturbation theory for linear operators. Berlin, Heidelberg, New York: Springer 1976

[Kr] Krein, S.G.: Linear differential equations in Banach spaces. Translations of Mathematical Monographs, Vol. 29. Providence RI: Amer. Math. Sci. 1971

[KS] Klein, M., Seiler, R.: Power-law corrections to the Kubo formula vanish in quantum Hall effect. Commun. Math. Phys. 128, 141-160 (1990)

[L] Lenard, A.: Adiabatic invariance to all orders. Ann. Phys. 6, 261-276 (1959)

[M] Messiah, A.: Quantum mechanics. II. Amsterdam: North-Holland 1969

[N1] Nenciu, G.: On the adiabatic theorem of quantum mechanics. J. Phys. A: Math. Gen. 13, L 15-L 18 (1980)

[N2] Nenciu, G.: Adiabatic theorem and spectral concentration. Commun. Math. Phys. 82, 125-135 (1981)

[N3] Nenciu, G.: Asymptotic invariant subspaces, adiabatic theorems and block diagonalisation. In: Boutet de Monvel et al. (eds.), Recent developments in quantum mechanics, pp. 133-149. Dordrecht: Kluver 1991

[N4] Nenciu, G.: Linear adiabatic theory and applications: exponential estimates. Preprint Univ. of Bucharest, 1991

[NR1] Nenciu, G., Rasche, G.: Adiabatic theorem and Gell-Mann-Low formula. H. P. A. 62, 372388 (1989)

[NR2] Nenciu, G., Rasche, G.: On the adiabatic theorem for non-self-adjoint hamiltonians. Preprint Univ. of Zürich, 1992

[T] Tanabe, H.: Equations of evolution. London: Pitman 1966 